\title{
The influence of DNA polymorphism of multidrug resistant 1 (MDR1) on the effect of midazolam pretreatment in children
}

\author{
Hyo-Jin Byon, Keun-Suk Park, Yong-Hee Park, Jin-Tae Kim, Chul-Woo Jung, and Hee-Soo Kim \\ Department of Anesthesiology and Pain Medicine, College of Medicine, Seoul National University, Seoul, Korea
}

Background: Patients showed a different response following intravenous midazolam injection. Some children showed irritability or were not sedated by midazolam. We hypothesized that there may be genetic variations of the MDR1 gene, based on the response to midazolam.

Methods: One hundred and ninety-three pediatric patients were recruited in this study. Midazolam (0.1 mg/kg) was injected intravenously before surgery. Anxiety score (activity, vocalizations, emotional expressivity, state of apparent arousal) was checked before and 5 minutes after midazolam injection. In addition, other manifestations after midazolam injection were recorded. After anesthesia, $2 \mathrm{ml}$ of blood was sampled. Children were genotyped MDR1. Haplotype was analyzed using the software package PHASE, version 2.0.

Results: The observed frequencies of MDR1 haplotype of TTT, TGC, CAC, CGC were $0.334,0.205,0.182$ and 0.225, respectively. There was no significant correlation between the response of midazolam and the MDR1 haplotype of TTT, TGC, CAC or CGC $(\mathrm{P}=0.98)$.

Conclusions: Genotyping of MDR1 may not be related to the response of midazolam in children. (Korean J Anesthesiol 2012; 62: 332-336)

Key Words: Genetic polymorphism, MDR1, Midazolam.

Received: May 31, 2011. Revised: 1st, July 8, 2011; 2nd, August 2, 2011; 3rd, August 10, 2011; 4th, August 23, 2011. Accepted: August 24, 2011. Corresponding author: Hee-Soo Kim, M.D., Department of Anesthesiology and Pain Medicine, College of Medicine, Seoul National University, 101, Daehak-ro, Jongno-gu, Seoul 110-744, Korea. Tel: 82-2-2072-3659, Fax: 82-2-745-5587, E-mail: dami0605@snu.ac.kr

(c) This is an open-access article distributed under the terms of the Creative Commons Attribution Non-Commercial License (http:// creativecommons.org/licenses/by-nc/3.0/), which permits unrestricted non-commercial use, distribution, and reproduction in any medium, provided the original work is properly cited. 


\section{Introduction}

Midazolam is a short-acting drug in the benzodiazepine class that is used for inducing sedation and amnesia before medical procedures, or anesthesia $[1,2]$. Sometimes, patients showed a paradoxical reaction such as crying, restlessness or vocalization after midazolam administration [3]. Kain et al. [4] reported that when children received midazolam as a premedication, some of them showed a paradoxical response and the degree of this response was related with the child's age or anxiety level. To explain this paradoxical reaction, a change in the activation of GABA receptors [5] or genetic variation of benzodiazepine receptors was suggested [6].

P-glycoprotein (permeability glycoprotein, P-pg) is a wellcharacterized ABC-transporter of the MDR/TAP subfamily. It is a $170 \mathrm{kDa}$ trans-membrane glycoprotein encoded by the multidrug resistance 1 (MDR1 or $\mathrm{ABCB} 1$ gene). The function of P-pg is absorption, distribution or elimination of drugs. There are more than 28 of genetic variants of P-pg [7]. Polymorphism of the MDR 1 gene can cause refractoriness of anti-epileptic drugs [8], or resistance to steroids in early nephritic syndromes [9]. Based on this, a patient's responses to midazolam correlates with MDR 1 gene polymorphism. In this study, we administered midazolam preoperatively, observed the patient response and searched for a relationship with MDR1 gene polymorphism.

\section{Materials and Methods}

After approval by the Institutional Ethics Committee, 193 American Society of Anesthesiologists (ASA) I-II infants and children, aged from 2 to 12 years undergoing strabismus surgery, were enrolled in this study. Informed consent was obtained from the parents of all infants and children. Exclusion criteria included ASA status III-V, upper respiratory infection within 4 weeks, history of neurologic disease or history of genetic disorder.

After fasting according to age (less than 36 months: 6 hours, more than 36 months: 8 hours), the patient arrived at the operating room without premedication. Before administration of midazolam, the patient was checked for consciousness. Every patient was clear and anxiety degree was measured by modified Yale preoperative anxiety scale (mYPAS, Table 1) before midazolam injection. Midazolam $0.1 \mathrm{mg} / \mathrm{kg}$ was administered and then a patient was observed for five minutes An anesthesiologist, who measured anxiety degree before injecting midazolam, measured and recorded anxiety degree again with mYPAS after injecting midazolam five minutes later. Sedation or anxiety degree was always measured with parents attending. After mYPAS was measured five minutes later, a patient was transferred to the operation room immediately. Then, electrocardiography, non-invasive blood pressure, oxygen saturation,

Table 1. Modified Yale Preoperative Anxiety Scale (mYPAS) Assessments

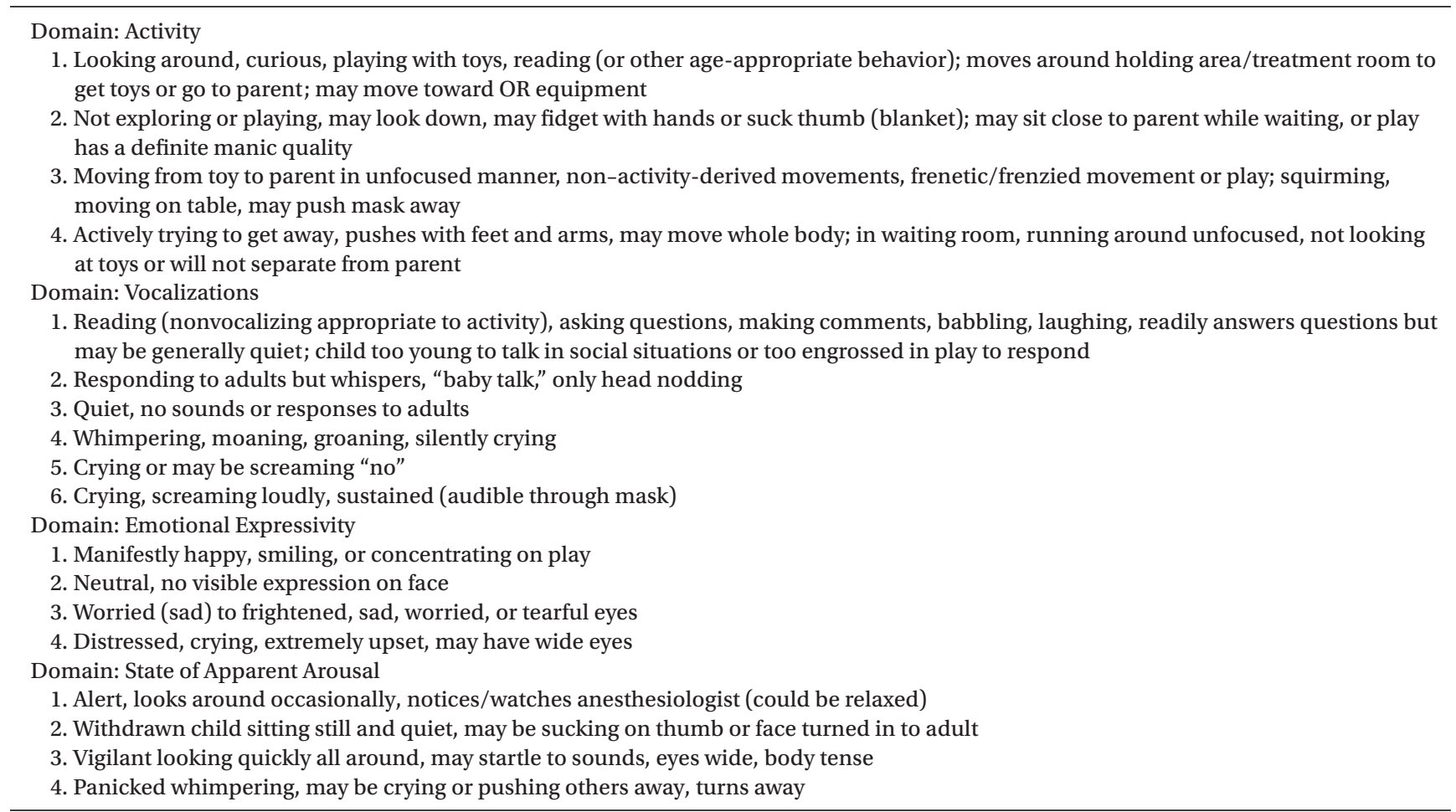


partial pressure of carbon dioxide and sevofluran concentration were monitored. Atropine $0.02 \mathrm{mg} / \mathrm{kg}$ (maximum dose $0.5 \mathrm{mg}$ ) were administered to every patient and then, thiopental $6 \mathrm{mg} /$ $\mathrm{kg}$ was administered intravenously. After losing consciousness, the patients were ventilated manually with 8 vol\% sevoflurane and $100 \%$ oxygen. For muscle relaxation, rocuronium $0.6 \mathrm{mg} /$ $\mathrm{kg}$ was administered. During the induction of anesthesia, 2 $\mathrm{ml}$ of venous blood was collected for measuring midazolam concentration and conducting a genotype test. The blood was stored in a vacuum EDTA tube at $-70^{\circ} \mathrm{C}$. After appropriate muscle relaxation was established, insertion of a larynx mask or endotracheal intubation was performed. Then, anesthesia was maintained with $2-3$ vol\% sevoflurane in $50 \%$ oxygen and $50 \%$ nitrous oxide. After surgery, muscle relaxation was reversed and a patient was transferred to the recovery room after extubation with complete awakening.

According to a previous study, a non-responder was decided when activity was more than 3 , vocalization was more than 4 , emotional expressivity was more than 3 and arousal was more than 3 in mYPAS [4]. When even one of four items was decided as a non-responder, the patient was decided as a non-responder group in this study. After administering midazolam, a case of other responses, which were not classified in mYPAS, were also recorded.

\section{DNA extraction}

General anesthesia was maintained using a conventional method and $2 \mathrm{ml}$ of venous blood was collected into a tube containing EDTA and stored at refrigeration temperatures. The sample was centrifuged for a genotype test and genomic DNA was extracted by QIAamp DNA Mini kit (Qiagen Inc., Valencia, CA) using the leukocyte layer. The blood sample was centrifuged at 2,500 rpm for 10 minutes and then the leukocyte layer was separated. In the leukocyte layer, $200 \mu \mathrm{l}$ was collected and mixed with QIAGEN protease $20 \mu \mathrm{l}$ and AL buffer 200 $\mu \mathrm{l}$, after which it was shaken by vortex mixer for 15 seconds. After incubation at $56^{\circ} \mathrm{C}$ for 10 minutes, the mixture was centrifuged. A total of $200 \mu \mathrm{l}$ of $96-100 \%$ ethanol was added and the sample was vortexed again for 15 seconds, after which it was centrifuged again at 2,500 rpm for 10 minute The mixture was transferred into QIAamp Spin Column and centrifuged at $13,000 \mathrm{rpm}$ for 1 minute. The DNA was then extracted according to the manufacturer's recommendation. The A260/A280 ratio of the DNA samples 1.7-1.9, which confirmed that they were relatively pure.

\section{Analysis of the genotype}

The method of Lindberg et al. [10] was used for DNA ampli- fication by polymerase chain reaction (PCR). Primers for amplification of the base sequences are shown in Table 2. The Total PCR reagent reaction volume was $25 \mu \mathrm{l}$, consisting of g-DNA $2 \mu \mathrm{l}$, Taq polymerase (Takara, Japan) $0.1 \mu \mathrm{l}$, dNTP $2 \mu \mathrm{l}$ (100 $\mathrm{uM}), 10 \times$ buffer $2.5 \mu \mathrm{l}$, AC D/W $17.4 \mu \mathrm{l}$, sense and antisense primers $0.5 \mu$ each. PCR conditions were as follows: $94^{\circ} \mathrm{C}$ and 5 minutes for pre-denaturation, followed by 30 cycles of $94^{\circ} \mathrm{C}$ denaturation for 30 seconds, 56 annealing ${ }^{\circ} \mathrm{C}$ for 30 seconds and $72{ }^{\circ} \mathrm{C}$ synthesis for 1 minute, and finally $72^{\circ} \mathrm{C}$ extension for 10 minutes. The genotype of MDR1 1236C > T (assay ID: C_7586662_10), ABCG2 421C > A (assay ID: C_15854163_70) and ABCC2 1249G > A (assay ID: C_22272980_20) SNPs was analyzed by established TaqMan Genotyping Assays (Applied Biosystems).

\section{Statistical analysis}

Alleles of modified single nucleotide polymorphism (SNP) and the frequency of a genotype were evaluated by Chi-square test with deviation from Hardy-Weinberg equilibrium. Statistical meanings of linkage disequilibrium between pairs of SNPs were evaluated by the Fischer's exact test. The result was expressed as number or percentage (\%), and SPSS program (SPSS Ver 19.0, Chicago, IL, USA) was used for all statistical analysis of the data. Logistic regression was used for correlation by each genotype in the responder and non-responder group after administering midazolam. Statistical significance was set as $\mathrm{P}<0.05$.

\section{Results}

A total of 193 patients were enrolled in this study. The average age was 7.5-years-old, the average height was $123.5 \mathrm{~cm}$ and the average weight was $27.6 \mathrm{~kg}$ (Table 3).

When anxiety degree was measured by mYPAS before administering midazolam, activity was more than 3 in 18 patients, vocalization was more than 4 in 21 patients, emotional expressivity was more than 3 in 35 patients and arousal was more than 3 in 15 patients. Twenty-five patients were applicable to two

Table 2. Demographic Data and Midazolam Plasma Concentration Levels

\begin{tabular}{lcc}
\hline & $\begin{array}{c}\text { Responder } \\
(\mathrm{N}=154)\end{array}$ & $\begin{array}{c}\text { Non-responder } \\
(\mathrm{N}=39)\end{array}$ \\
\hline $\mathrm{M}: \mathrm{F}$ & $80: 74$ & $18: 21$ \\
Age $(\mathrm{yr})$ & $7.2 \pm 2.6$ & $7.6 \pm 2.9$ \\
$\mathrm{~B} \mathrm{wt}(\mathrm{kg})$ & $25.8 \pm 10.4$ & $28.9 \pm 9.9$ \\
$\mathrm{Ht}(\mathrm{cm})$ & $122.7 \pm 19.2$ & $124.6 \pm 19.4$ \\
Midazolam plasma & $83.4 \pm 31.5$ & $81.6 \pm 34.2$ \\
concentration $(\mathrm{ng} / \mathrm{ml})$ & & \\
\hline
\end{tabular}

Values are means \pm SD or numbers. 
Table 3. Primer and Probe Sequences for MDR1 2677G $>$ T, 2677G $>$ A, 3435C $>$ T

\begin{tabular}{clc}
\hline SNP & \multicolumn{1}{c}{ Primers } & Probe \\
\hline MDR1 2677G $>$ T & 5'-GAAATGAAAATGTTGTCTGGACAAGCA-3' & VIC-TTCCCAGCACCTTC \\
MDR1 2677G > A & 5'-CTTAGAGCATAGTAAGCAGTAGGGAGT-3' & FAM-TTCCCAGAACCTTC \\
MDR1 3455C $>$ T & 5'-GAAATGAAAATGTTGTCTGGACAAGCA-3 & VIC-TTCCCAGCACCTTC \\
& 5'-CTTAGAGCATAGTAAGCAGTAGGGAGT-3 & FAM-CTTCCCAGTACCTTC \\
& 5'-CTGTTTGACTGCAGCATTGCT-3' & VIC-CCCTCACGATCTCTT \\
5'-ATGTATGTTGGCCTCCTTTGCT-3' & FAM-CCCTCACAATCTCTT \\
\hline
\end{tabular}

Table 4. Incidence of Non-responder in the Study Subjects

\begin{tabular}{lcc}
\hline & $\begin{array}{c}\text { Before } \\
\text { midazolam }\end{array}$ & $\begin{array}{c}5 \text { minutes after } \\
\text { midazolam injection }\end{array}$ \\
\hline Activity $\geq 3$ & $18(9.3)$ & $21(10.8)$ \\
Vocalization $\geq 4$ & $21(10.8)$ & $13(6.7)$ \\
Emotional Expressivity $\geq 3$ & $35(18.1)$ & $16(8.2)$ \\
Arousal $\geq 3$ & $15(7.7)$ & $17(8.8)$ \\
\hline
\end{tabular}

Values are number (\% of total patients).

items or more and their average age was 6.8-years-old (Table 4).

After administering midazolam, a total of 39 patients showed anxiety and excitement (20.2\%). Activity was more than 3 in 21 patients, vocalization was more than 4 in 13 patients, emotional expressivity was more than 3 in 16 patients and arousal was more than 3 in 17 patients. Eighteen patients were applicable to two items or more (Table 4 ).

Continuously high scores before and after administering midazolam were shown in 4 patients for activity, 4 patients for vocalization, 2 patients for emotional expressivity and in 4 patients for arousal. Among them, 5 patients were classified into the non-responder group (5, 7, 8, 9, and 12-years-old). Stable states before administering and high scores after administering midazolam were shown in 13 patients for activity, 14 patients for vocalization, 8 patients for emotional expressivity and in 10 patients for arousal.

After administrating midazolam, 43 patients showed smiling or laugh and no patient among them was classified into the non-responder group.

To measure the concentration of midazolam, blood samples were collected after inducing anesthesia. The average concentration was $83.4 \mathrm{ng} / \mathrm{ml}$ in the responder group and $81.6 \mathrm{ng} /$ $\mathrm{ml}$ in the non-responder group. For estimation of a haplotype in three genotypes of 1236C $>\mathrm{T}, 2677 \mathrm{G}>\mathrm{T} / \mathrm{A}, 3435 \mathrm{C}>\mathrm{T}$ in MDR-1 gene, TGC, CAC, CGC, TTT were mainly generated and the frequency in each group was shown in Table 5.

When midazolam was administered as pre-treatment of anesthesia to children between 2 and 12 years old with strabismus surgery, a total of 39 patients $(20.2 \%)$ showed anxiety and excitement without sedation. Genetic polymorphism and adverse reactions after administrating midazolam were not correlated ( $\mathrm{P}=0.98$ or 0.99$)$.
Table 5. Numbers and Frequencies of MDR1 Haplotype

\begin{tabular}{cccc}
\hline & Responder & Nonresponder & \\
\cline { 2 - 3 } & Number $(\%)$ & Number $(\%)$ & \\
\hline CAC & $55(0.182)$ & $13(0.169)$ & 0.99 \\
CGC & $68(0.225)$ & $13(0.169)$ & 0.98 \\
TGC & $62(0.205)$ & $17(0.221)$ & 0.99 \\
TTT & $101(0.334)$ & $28(0.364)$ & 0.99 \\
\hline
\end{tabular}

\section{Discussion}

Midazolam is commonly used for premedication of anesthesia or as sedative in children. When it is used in the clinical field, an unexpected reaction can sometimes occur. According to a previous study, only $86 \%$ of children administered with midazolam before anesthesia showed appropriate sedation for mask ventilation [11]. In other studies, $14.1 \%$ of children, who were administered with $0.5 \mathrm{mg}$ of midazolam, showed severe anxiety and excitement but the rate was $20.2 \%$ in this clinical study so it was more than previous studies [4]. Kain et al. applied 0.5 $\mathrm{mg} / \mathrm{kg}$ of midazolam per oral but $0.1 \mathrm{mg} / \mathrm{kg}$ of midazolam was injected intravenously in this study and the standards to classify the responder and non-responder group were different so this may cause the difference in the results. In addition, Kain et al. reported that adverse reactions of midazolam increased as a patient was more emotional, more worried and younger. However, the average age of patients, who showed high scores in mYPAS before administrating midazolam, was not low and 5 of them were classified into the non-responder group after administrating midazolam in this study. The age of each patient was evenly distributed so it was different to the previous studies. The difference might be caused by the difference of the standards to classify after administering a drug and observing. In other words, a patient was classified by scores of every item after oral administration time after time in previous studies but a patient was classified into a non-responder group when he (or she) was applicable to even one item after measuring scores for each item in this study, which may lead to the difference.

Toll-Sander et al. [12] studied the relationship between midazolam and p-glycoprotein and reported that midazolam was very permeable in p-glycoprotein substrate. Therefore, 
p-glycoprotein is important to show the effect of midazolam and genetic polymorphism of p-glycoprotein was assumed to show the difference in the effect of midazolam but was not confirmed in this study. If the role of p-glycoprotein did not crucially change despite the genetic polymorphism, the difference in clinical signs after administering midazolam could not be explained. For this reason, further studies are required.

Walbergh et al. [13] reported that the maximum effect of midazolam was shown in 11 minutes when $0.1 \mathrm{mg} / \mathrm{kg}$ midazolam was administered through the nasal cavity and the average blood concentration was $72.2 \mathrm{ng} / \mathrm{ml}$. In our study, the average blood concentration was $80 \mathrm{ng} / \mathrm{ml}$. The blood was collected 6 minutes later after administering midazolam so the concentration may have been higher than in previous reports. Intravenous injection could be also a factor for the difference. However, the concentration of midazolam between the responder and nonresponder group was not different so a different reaction by the blood concentration was difficult to explain.

The most common reaction was a laugh in adverse reactions after administrating a drug and the specificity of genetic polymorphism was not found. Although the specificity was found, it was not harmful or significant clinically. Therefore, polymorphism of other genes was not needed.

In this clinical study, there were several limitations. Other genetic polymorphisms could be predicted although it was not studied in this study. A receptor of midazolam is GABAA receptor and $\gamma 2$ subunit is essential for modification of binding sites. In previous studies, a sleeping effect of benzodiazepine did not change but anxiety was higher when $\gamma 2$ subunit of GABAA was not present [14]. Therefore, genetic polymorphism of GABRG2, which encodes $\gamma 2$ subunit of the GABAA receptor, should be studied. The other limitation was a standard of classification, which when changed led to a correlation between genetic polymorphism and reactions for midazolam.

To conclude, when anxiety was measured by mYAS after intravenous injection of $0.1 \mathrm{mg} / \mathrm{kg}$ midazolam, genetic polymorphism of MDR1 was not found in the non-responder group compared to the responder group. However, further studies are required to find correlations with other genetic polymorphisms.

\section{Acknowledgements}

This study is supported by Seoul National University Hospital Grant (0420080990).

\section{References}

1. Bae JH, Koo BW, Kim S, Lee D, Lee ET, Kang C. The effects of midazolam administered postoperatively on emergence agitation in pediatric strabismus surgery. Korean J Anesthesiol 2010; 58: 45-9.

2. Kain ZN, Caldwell-Andrews AA, Maranets I, McClain B, Gaal D, Mayes LC, et al. Preoperative anxiety and emergence delirium and postoperative maladaptive behaviors. Anesth Analg 2004; 99: 164854.

3. Golparvar M, Saghaei M, Sajedi P, Razavi SS. Paradoxical reaction following intravenous midazolam premedication in pediatric patients - a randomized placebo controlled trial of ketamine for rapid tranquilization. Paediatr Anaesth 2004; 14: 924-30.

4. Kain ZN, MacLaren J, McClain BC, Saadat H, Wang SM, Mayes LC, et al. Effects of age and emotionality on the effectiveness of midazolam administered preoperatively to children. Anesthesiology 2007; 107: 545-52.

5. Hall RC, Zisook S. Paradoxical reactions to benzodiazepines. Br J Clin Pharmacol 1981; 11 Suppl 1: 99S-104S.

6. Short TG, Forrest P, Galletly DC. Paradoxical reactions to benzodiazepines--a genetically determined phenomenon? Anaesth Intensive Care 1987; 15: 330-1.

7. Eichelbaum M, Fromm MF, Schwab M. Clinical aspects of the MDR1 (ABCB1) gene polymorphism. Ther Drug Monit 2004; 26: 180-5.

8. Lazarowski A, Czornyj L. Potential role of multidrug resistant proteins in refractory epilepsy and antiepileptic drugs interactions. Drug Metabol Drug Interact 2011; 26: 21-6.

9. Choi HJ, Cho HY, Ro H, Lee SH, Han KH, Lee H, et al. Polymorphisms of the MDR1 and MIF genes in children with nephrotic syndrome. Pediatr Nephrol 2011; 26: 1981-8.

10. Lindberg AM, Polacek C, Johansson S. Amplification and cloning of complete enterovirus genomes by long distance PCR. J Virol Methods 1997; 65: 191-9.

11. Cote CJ, Cohen IT, Suresh S, Rabb M, Rose JB, Weldon BC, et al. A comparison of three doses of a commercially prepared oral midazolam syrup in children. Anesth Analg 2002; 94: 37-43.

12. Tolle-Sander S, Rautio J, Wring S, Polli JW, Polli JE. Midazolam exhibits characteristics of a highly permeable P-glycoprotein substrate. Pharm Res 2003; 20: 757-64.

13. Walbergh EJ, Wills RJ, Eckhert J. Plasma concentrations of midazolam in children following intranasal administration. Anesthesiology 1991; 74: 233-5.

14. Chandra D, Korpi ER, Miralles CP, De Blas AL, Homanics GE. GABAA receptor gamma 2 subunit knockdown mice have enhanced anxietylike behavior but unaltered hypnotic response to benzodiazepines. BMC Neurosci 2005; 6: 30. 